J. Clin. Chem. Clin. Biochem.

Vol. 17, 1979, pp. 721-724

\title{
Lichtempfindlichkeit der Kreatinkinase in Kontrollseren
}

\author{
Von G. Weidemann, R. D. Schmid und L. Reichold \\ Institut für Klinische Chemie des Klinikums der Stadt Nürnberg
}

(Eingegangen am 25. Oktober 1978/22. Februar 1979)

Dem Gedenken an Professor Dr. Gábor Szász gewidmet ${ }^{1}$ )

Zusammenfassung: In den verschiedenen kommerziellen Kontrollseren ist die Kreatinkinase unterschiedlich lichtempfindlich;

In 12 von 22 Kontrollseren, abgefüllt in Reaktionsgefäße und $4 \mathrm{~h}$ bei $25^{\circ} \mathrm{C}$ einer Lichtquelle ausgesetzt, deren Intensität der normalen Laborbeleuchtung entspricht, nimmt die katalytische Konzentration der Kreatinkinase um 25-63\% ab. Unter den gleichen Bedingungen ist die Kreatinkinase in Patientenseren stabil. Bei intensiverer Belichtung nimmt die Instabilität der Kreatinkinase in den „lichtempfindlichen Kontrollseren“ zu; bei Sonnenlichtexposition erwies sich die Kreatinkinase in allen untersuchten Kontroll- und Patientenseren als lichtempfindlich. Mercaptoethanol-Zusatz stabilisiert die Kreatinkinase in den ,lichtempfindlichen Kontrollseren“". Die Ursache für die unterschiedliche Lichtempfindlichkeit der verschiedenen Kontrollseren ist noch unklar.

\section{Light sensitivity of creatine kinase in control sera}

Summary: The light sensitivity of creatine kinase in different control sera was investigated. Control sera were dispensed into reaction vessels, then exposed for $4 \mathrm{~h}$ at $25^{\circ} \mathrm{C}$ to a light source equivalent in intensity to normal laboratory illumination. In 12 out of 22 control sera, the catalytic concentration of the creatine kinase fell by $25-63 \%$. Under the same conditions, in patient sera, creatine kinase is stable. In the "light sensitive" control sera, the instability of the enzyme increased with the intensity of illumination. When exposed to sunlight, creatine kinase was unstable in all the investigated control and patient sera. The addition of mercaptoethanol stabilized the creatine kinase in "light sensitive" control sera. The reason for the different light sensitivity of different control sera is not known.

\section{Einführung}

Die Instabilität der Kreatinkinase in Kontrollseren, auf die Szász (1) bereits 1970 und Hetland \& Lund (2) kürzlich erneut hingewiesen haben, ist nach unseren Erfahrungen u.a. auf die unterschiedliche Lichtempfindlichkeit des Enzyms in den verschiedenen Kontrollseren zurückzuführen. Im folgenden wird über den bisher kaum beachteten Einfluß von Licht auf die Kreatinkinase in kommerziellen Kontrollproben sowie in Patientenseren berịchtet.

\section{Material und Methoden}

Material

Monotest $10 \mathrm{CK}$ NAC aktiviert, Fa. Boehringer Mannheim.

\section{Kontrollproben}

Ledernorm, $\bar{F}$ a Cyanamid; Kontrollogen E,-L,-LP, Fa. Behringwerke; Precinorm E, Precipath E, Precinorm U, Fa. Boehringer Mannheim; Validate A,-N, Verșatol E, Fa. Gödecke, Europa- kontrolle I, -II, Fa. Hyland; Enzatrol, Moni-Trol I, -II, Fa. Merz und Dade; Pathonorm H, -L, Seronorm, Fa. Nyegaard; Kontrollserum N, -P, Fa. Roche;

Kreatinkinase aus Kaninchenmuskel (126969), Fa. Boehringer Mannheim; Rinderserumalbumin, reinst, Behringwerke.

\section{Einwegartikel}

Reaktionsgefäße $3810 \mathrm{Fa}$. Eppendorf; LKB-Proberöhrchen (Polypropylen und Polystyrol), Fa. Greiner; LKB-Küvetten (Acryl), Fa. Sarstedt; Serumcups (Polypropylen), Fa. Gilford.

\section{Methoden}

Die Kreatinkinase-Bestimmung erfolgte mit dem Analysensystem $3500, \mathrm{Fa}$. Gilford; Messung bei $25^{\circ} \mathrm{C}$ und $340 \mathrm{~nm}$; Vorinkubation: $5 \mathrm{~min}$.

Präżisisionskontrolle der Kreatinkinase-Bestimmung Präzision in der Serie bei

a) normaler kataly tischer Kreatinkinase-Konzentration $\mathrm{n}=20$

$\overline{\mathrm{x}}=51 \mathrm{U} / 1$

$s=0,85 \mathrm{U} / 1$

VK $=1,66 \%$

\footnotetext{
1) Der żu einem früheren Zeitpunkt vorgesehene Abdruck der Arbeit wurde auf Wunsch der Autoren für dieses spezielle Heft zurückgesteilt.
} 
b) erhöhter katalytischer Kreatinkinase-Konzentration

$$
\begin{aligned}
& \mathrm{n}=20 \\
& \overline{\mathrm{x}}=238 \mathrm{U} / 1 \\
& \mathrm{~s}=1,04 \mathrm{U} / 1 \\
& \mathrm{VK}=0,44 \%
\end{aligned}
$$

Präzision von Tag zu Tag während der Versuchsdauer

$$
\begin{aligned}
& \bar{x}=128 \mathrm{U} / 1 \\
& \mathrm{~s}=2,3 \mathrm{U} / 1 \\
& \mathrm{Vk}=1,8 \%
\end{aligned}
$$

Die Kontrollproben wurden 30 min im Dunkeln bei Raumtemperatur rekonstitutioniert.

Die Belichtung der Kontrollproben bzw. Patientenseren erfolgte in Einweg-Reaktionsgefäßen und -küvetten bzw. in den Originalfläschchen vor einem Lichtkasten $(24 \times 26 \mathrm{~cm})$, bestückt mit drei $40 \mathrm{~cm}$ langen Leuchtstoffröhren (15 W, Farbton "Coolwhite"), abgedeckt mit einer $0,4 \mathrm{~cm}$ dicken Milchglasscheibe. Abstand der Proben zur Lichtquelle: $23 \mathrm{~cm}$ entsprechend der Helligkeit eines gut beleuchteten Arbeitsplatzes im Labor $=$ "normale Belichtung". Für erhöhte Lichtintensität wurde der Abstand auf $1 \mathrm{~cm}$ verringert = "intensivere Belichtung". Außerdem wurden die Kontroll- und Patientenseren dem Sonnenlicht ausgesetzt $=$ "Sonnenlicht". Die elektrophoretische Trennung der Isoenzyme der Kreatinkinase erfolgte in Agargel nach einer eigenen bisher nicht publizierten Methode.

\section{Ergebnisse und Diskussion}

1. Die Kontrollseren wurden in den Originalfläschchen, bzw. wie die Patientenseren in Reaktionsgefäße abgefüllt, zunächst einer künstlichen Lichtquelle ausgèsètzt, deren Intensität der Helligkeit eines gut ausgeleuchte= ten Arbeitsplatzes im Laboratorium entspricht.

Tabelle 1 zeigt die Lichtempfindlichkeit der Kreatinkinase in verschiedenen Kontrollseren nach $4 \mathrm{~h}$ "normaler Belichtung": Im Vergleich zu den bei $4^{\circ} \mathrm{C}$ und $25^{\circ} \mathrm{C}$ dunkel aufbewahrten Kontrollseren findet man bei $4{ }^{\circ} \mathrm{C}$ in 11 , bei $25^{\circ} \mathrm{C}$ in 12 von 22 Kontrollseren eine Abnahme der katalytischen Konzentration der Kreatinkinase von $25 \%$ bis $63 \%$. Im folgenden werden die Kontrollseren, bei denen die katalytische Konzentration durch $4 \mathrm{~h}$, normale Belichtung " um mehr als $25 \% \mathrm{ab}$ nimmt, als „lichtempfindliche Kontrollseren“ bezeichnet. Unter diesen fällt die erhöhte Lichtempfindlichkeit der Kreatinkinase im Precinorm $E$ und Precipath $E$ bei $25^{\circ} \mathrm{C}$ gegenüber $4{ }^{\circ} \mathrm{C}$ auf.

Die Abnahme der katalytischen Konzentration der Kreatinkinase in Abhängigkeit von der Belichtungs-

\begin{tabular}{|c|c|c|c|c|c|c|}
\hline \multirow[t]{2}{*}{ Kontrollserum } & \multicolumn{3}{|c|}{ dunkel } & \multicolumn{2}{|c|}{ belichtet } & \multirow[b]{2}{*}{$\begin{array}{l}\text { Sollwert } \\
\text { U/1 }\end{array}$} \\
\hline & $\begin{array}{l}4^{\circ} \mathrm{C} \\
\mathrm{U} / 1\end{array}$ & & $\begin{array}{l}25^{\circ} \mathrm{C} \\
\mathrm{U} / 1\end{array}$ & $\begin{array}{l}4^{\circ} \mathrm{C} \\
\mathrm{U} / 1\end{array}$ & $\begin{array}{l}25^{\circ} \mathrm{C} \\
\mathrm{U} / 1\end{array}$ & \\
\hline Ledernorm $2905-428$ & 18 & & 21 & 17 & 18 & $* *$ \\
\hline Kontrollogen E 2102 & 288 & & 293 & 275 & 281 & 297 \\
\hline Kontrollogen L $447 \mathrm{C}$ & 52 & & 57 & 42 & 43 & $* *$ \\
\hline Kontrollogen LP $3202 \mathrm{~F}$ & 192 & & 207 & $131 *$ & $137 *$ & $* *$ \\
\hline Precinorm E 652 & 121 & & 122 & 104 & $87 *$ & 119 \\
\hline Precipath E 628 & 109 & & 109 & $76 *$ & $40 *$ & 107 \\
\hline Precipath E 629 & 90 & & 87 & $67 *$ & $33 *$ & 88 \\
\hline Precinorm U 609 & 122 & & 129 & 115 & 118 & 117 \\
\hline Precinorm U 722 & 33 & & 36 & 30 & 34 & 35 \\
\hline Validate A 0433067 B & 165 & & 171 & $112 *$ & $124 *$ & 162 \\
\hline Validate N 0527087 & 58 & & 55 & $41^{*}$ & $39 *$ & 53 \\
\hline Versatol E 4 B 842 & 57 & & 53 & 58 & 58 & $* *$ \\
\hline Europa-Kontrolle I N O 4 & 80 & & 76 & $45^{*}$ & $46 *$ & $* *$ \\
\hline Europa-Kontrolle II P 11 & 167 & & 150 & $100 *$ & $97 *$ & 149 \\
\hline Enzatrol 245 & 230 & & 225 & 205 & 201 & 217 \\
\hline Moni-Trol I 147 A & 75 & $\cdots$ & 77 & $37 *$ & $40 *$ & 79 \\
\hline Moni-Trol II 51 B & 159 & & 169 & 94* & $92 *$ & 175 \\
\hline Pathonorm H 12 & 321 & & 324 & $226 *$ & $240 *$ & $* *$ \\
\hline Pathonorm L 12 & 52 & & 57 & 42 & 43 & $* *$ \\
\hline Seronorm 128 & 127 & . & 121 & $77^{*}$ & $73^{*}$ & $* *$ \\
\hline Kontrollserum N Roche 1639 & 128 & & 133 & 122 & 117 & 124 \\
\hline Kontrollserum P Roche 2739 & 358 & & 365 & 334 & $331 \ldots$ & 379 \\
\hline
\end{tabular}

Tab. 1. Lichtempfindlichkeit der Kreatinkinase in verschiedenen Kontrollseren. Die Kontrollseren wurden $4 \mathrm{~h}$ bei $4{ }^{\circ} \mathrm{C}$ und $25^{\circ} \mathrm{C}$ im Dunkeln gehalten bzw. der ,normalen Belichtung“ ausgesetzt. Jeweils Mittelwert aus Doppelbestimmungen; zur Präzision der Kreatinkinase Bestimmung s. Methodik.

* im Vergleich zu dem bei entsprechender Temperatur dunkel aufbewahrten Kontrollserum Abnahme der katalytischen Konzentration über $25 \%=$,lichtempfindliches Kontrollserum".

** keune Angabe von Sollwerten für die N-Acetylcystein-aktivierte Kreatinkinase-Bestimmung. 
dauer ist aus Abbildung 1 ersichtlich. Die ,lichtempfindliche Kontrollprobe" Moni-Trol II wurde außerdem bei $20^{\circ} \mathrm{C}$ im Wechsel $30 \mathrm{~min}$ der ,normalen Belichtung" ausgesetzt und anschließend $30 \mathrm{~min}$. im Dunkeln gehalten. Im Gegensatz zu den in-vitroVersuchen von Morin (3) mit isolierter Kreatinkinase MB in hitzeinaktivierten Humanseren war während der Dunkelperiode in der Kontrollprobe, die ebenfalls Kreatinkinase MB enthält, keine partielle Reaktivierung der Kreatinkinase nachweisbar. Die Kontrollpfoben wurden in Einwegreaktionsgefäßen bzw. -küvetten aus Polystyrol, Polypropylen und Acryl sowie in den Originalfläschchen belichtet. Hierbei zeigte sịch, dạß die Art des Kunststoffmaterials keinen Einfluß auf das Ausmaß der Inaktivierung hatte. Erfolgte die Belliohtung der Kontrollproben in den Originalffäsẹehen, nahm die katalytische Konzentration der Kreatinkinase dagegen nicht oder deutlich geringer ạb (Tab. 2). Da auch einige

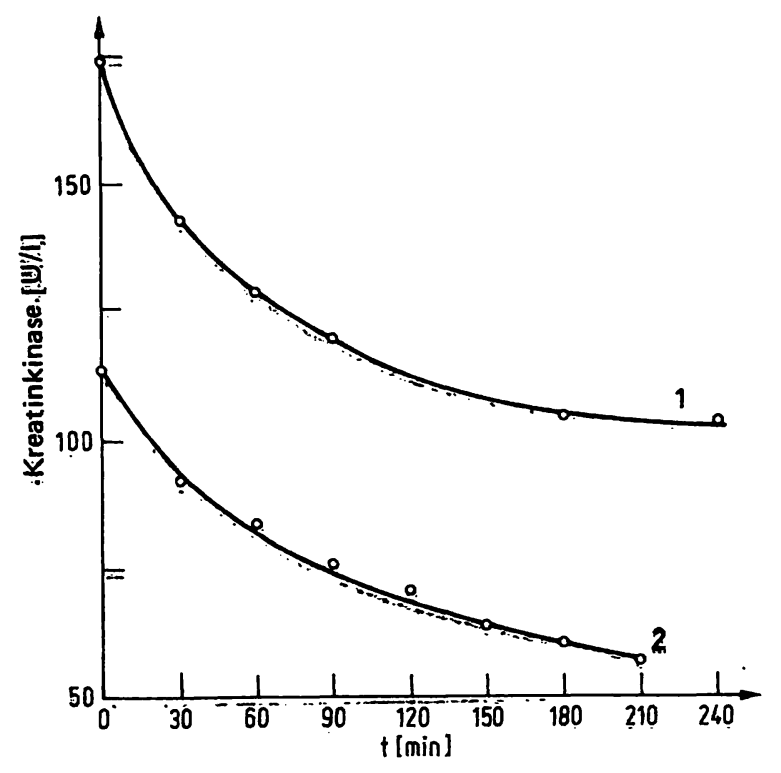

Abbb. 1. Abnahme def katalytischen Konzentration der Kreatinkinase bẹi ,normaler Belichtung"! und $25^{\circ} \mathrm{C}$ in Abhängigkeit von der Zeit. 1 = Moni=Trol II (51B), 2 = Precipạth E (620):

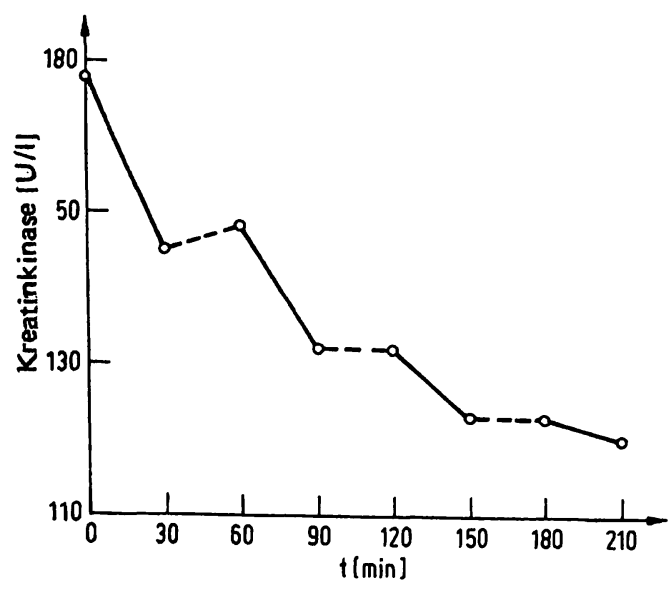

Abb. 2. Einfluß von Hell-Dunkel-Perioden auf die Stabilität der Kreatinkinase in Moni-Trol II (51 B). Moni-Trol II wurde in einem Eppendorf-Reaktionsgefäß bei $20^{\circ} \mathrm{C}$ im Wechsel $30 \mathrm{~min}$ der "normalen Belichtung" ausgesetzt, anschließend $30 \mathrm{~min}$ im Dunkeln gehalten. - Belichtung; - - - Dunkelperiode.

braun gefärbte Flaschen die Kreatinkinase nicht vollständig vor Inaktivierung durch Licht schützen, ist im Einzelfall zu prüfen, welche Kontrollseren im Dunkeln rekonstitutioniert werden müssen.

In Patientenseren, die unter analogen Bedingungen wie die Kontrollproben belichtet wurden, war die Kreatinkinase stabil. Es wurden 86 Proben untersucht, von denen 33 eine katalytische Konzentration der Kreatinkinase von 100-750 U/1 enthielten:

$\mathrm{x}=$ Ergebnisse der $4 \mathrm{~h}$ bei $4^{\circ} \mathrm{C}$ im Dunkeln aufbewahrten Seren

$\mathrm{y}=$ Ergebnisse der $4 \mathrm{~h}$ bei $25^{\circ} \mathrm{C}$ belichteten Proben

$y=1,004 x+0,245(U / 1)$

$r=0,999 s_{x y}=5,16$

2. In den ,lichtempfindlichen Kontrollseren " nimmt die Instabilität der Kreatinkinase zu, wenn die Intensität der künstlichen Belichtung erhöht bzw. mit direktem Sonnenlicht belichtet wird (Tab. 3). Außerdem zeigt die Tabelle 3 am Beispiel von Precinorm U und $5 \mathrm{~Pa}$ -

TTab. 2. "Normale Belichtung" der Konţollserenen bei $25^{\circ} \mathrm{C}$ in Reaktionsgefäßen aus verschiedenem Kunststoffmaterial sowie in den Originalfịäșchchen. J̣eweils Mittelwert aụs Doppelbestimmungen; zur Präzision der Kreatinkinase-Bestimmung s. Mcthodik.

\begin{tabular}{|c|c|c|c|c|c|}
\hline Sollwert & $\begin{array}{l}\text { Valldate A } \\
0433067 \mathrm{~B} \\
\mathrm{U} / 1 \\
162\end{array}$ & $\begin{array}{l}\text { Moni-Trol II } \\
51 \mathrm{~B} \\
\mathrm{U} / 1 \\
175\end{array}$ & $\begin{array}{l}\text { Moni-Trol I } \\
147 \text { B } \\
\text { U/1 } \\
79\end{array}$ & $\begin{array}{l}\text { Precipath E } \\
629 \\
\mathrm{U} / 1 \\
88\end{array}$ & $\begin{array}{l}\text { Europakontrolle II } \\
\text { P } 11 \\
\text { U/1 } \\
149\end{array}$ \\
\hline Belichtung in: Polypropylen (Eppendorf) & 117 & 104 & 44 & 51 & 90 \\
\hline Polypropylen (G̣reinẹr) & 118 & 106 & 44 & 51 & 96 \\
\hline Polypropylen (Gilford) & 124 & 92 & 40 & 53 & 97 \\
\hline Palysstyral (Greiner) & 121 & 109 & 43 & 50 & 96 \\
\hline Acryyl (Sarsțedț) & 124 & 103 & 45 & 49 & 97 \\
\hline Originalfläschchen (Farbe) & 167 (braun) & 158 (braun) & 58 (weiß) & 74 (braun) & 118 (braun) \\
\hline
\end{tabular}


tientenseren, daß die Kreatinkinase durch Sonnenlichtexposition auch in einer bei „Normalbelichtung" stabilen Kontrollprobe und, wie bereits von Thompson (4) mitgeteilt, in Patientenseren inaktiviert wird.

3. Die Ursache für die Instabilität der Kreatinkinase in Kontrollproben durch Lichteinwirkung ist noch unklar. Wie wir feststellten, wird durch Zusatz von Mercaptoethanol, $30 \mathrm{mmol} / \mathrm{l}$, zu den Kontrollproben vor der ,normalen Belichtung “ die Ǩreatinkinase vor Inaktivierung geschützt (Tab. 4); durch 30 min Inkubation der ,lichtempfindlichen Kontrollproben “ mit Mercaptoäthanol nach der Belichtung wird die Kreatinkinase nicht mehr reaktiviert. Wie weitere Untersuchungen ergaben, ist gereinigte Kreatinkinase aus
Kaninchenmuskel in Humanseren gelöst lichtempfindlicher als in Rinderserumalbumin. Auffällig hierbei ist, daß die Kreatinkinase in den einzelnen Humanseren unterschiedlich stark inaktiviert wird. Nach elektrophoretischen Untersuchungen der belichteten Kontrollseren sind alle Isoenzyme der Kreatinkinase lichtempfindlich. Möglicherweise enthalten die einzelnen Kontrollseren in unterschiedlicher Konzentration Bestandteile wie SH-gruppenhaltige Substanzen, die die Kreatinkinase stabilisieren. Andererseits kann nicht ausgeschlossen werden, daß Zusätze zu den Kontrollseren die Inștabilität der Kreatinkinase bei Lichteinwirkung erhöhen.

Tab. 3. Einfluß der Intensität der Belichtung auf die katalytische Konzentration der Kreatinkinase in Kontroll- und Patientenseren. Die Proben wurden in Eppendorf-Reaktionsgefäßen $4 \mathrm{~h}$ bei $20^{\circ} \mathrm{C}$ verschiedener Lichtintensität ạusgesetzt bzw. im Dunkeln gehalten. Jeweils Mittelwert aus Doppelbestimmungen; zur Präzision der Kreatinkinase-Bestimmung s. Methodik.

\begin{tabular}{|c|c|c|c|c|}
\hline Kontroll- bzw. Patientenserum & $\begin{array}{l}\text { "normale } \\
\text { Belichtung" } \\
\text { U/1 }\end{array}$ & $\begin{array}{l}\text {,intensinsive } \\
\text { Belịhtung" } \\
\text { U/1 }\end{array}$ & $\begin{array}{l}\text { „Sonnenlicht“" } \\
\text { U/1 }\end{array}$ & $\begin{array}{l}\text { dunkel } \\
\mathrm{U} / 1\end{array}$ \\
\hline $\begin{array}{l}\text { Moni-Trol II } 51 \text { B } \\
\text { Precipath E } 629 \\
\text { Precinorm U } 609 \\
\text { Pat. Nr. 1 } \\
\text { Pat. Nr. } 2 \\
\text { Pat. Nr. } 3 \\
\text { Pat. Nr. } 4 \\
\text { Pat. Nr. } 5\end{array}$ & $\begin{array}{r}97 \\
50 \\
115 \\
124 \\
293 \\
23 \\
397 \\
213\end{array}$ & $\begin{array}{r}85 \\
24 \\
110 \\
118 \\
289 \\
23 \\
382 \\
182\end{array}$ & $\begin{array}{r}49 \\
7 \\
95 \\
80 \\
176 \\
14 \\
282 \\
148\end{array}$ & $\begin{array}{r}171 \\
88 \\
118 \\
123 \\
291 \\
24 \\
391 \\
213\end{array}$ \\
\hline
\end{tabular}

Tab. 4. Stabilität der Kreatinkinase in Kontrollseren im Dunkeln bzw. bei „normaler Belichtung“ in Gegenwart von Mercaptoethanol. Die Seren wurden ohne und mit Zusatz von $30 \mathrm{mmol} / 1$ Mercaptoethanol $4 \mathrm{~h}$ bei $25^{\circ} \mathrm{C}$ der "normalen Belichtung " ausgesetzt bzw. im Dunkeln gehalten. Jeweils Mittelwert aus Doppelbestimmungen; zur Präzision der Kreatinkinase-Bestimmung S. Methodik.

\begin{tabular}{|c|c|c|c|c|}
\hline Kontrollserum & $\begin{array}{l}\text { dunkel } \\
\text { + Mercaptoethanol } \\
\mathrm{U} / 1\end{array}$ & $\begin{array}{c}\text { dunkel } \\
\text { U/1 }\end{array}$ & $\begin{array}{l}\text { „Bnormale } \\
\text { Belichtung“ } \\
\text { U/1 }\end{array}$ & $\begin{array}{l}\text { "normale } \\
\text { Belichtung“" } \\
+ \text { Mercaptoethano } \\
\text { U/1 }\end{array}$ \\
\hline $\begin{array}{l}\text { Moni-Trol II 51B } \\
\text { Precipath E } 629 \\
\text { Kontrollserum Roche P } 2739\end{array}$ & $\begin{array}{r}176 \\
87 \\
375\end{array}$ & $\begin{array}{r}208 \\
89 \\
375\end{array}$ & $\begin{array}{r}103 \\
50 \\
319\end{array}$ & $\begin{array}{r}215 \\
89 \\
366\end{array}$ \\
\hline
\end{tabular}

\footnotetext{
Addendum

Ähnliche Ergebnisse wie in der vorliegenden Arbeit wurden kürzlich von $B$. Perry et al. in einer Publikation „Effect of Light and Temperature on the Stability of Creatine Kinase in Human Sera and Controls" in Clin. Chem. 25 (4) 625-628 (1979) mitgeteilt.
}

\section{Literatur}

1. Szasz, G. (1970), diese Z. 8, 208-211

2. Hetland, O. \& Lund, P. K., (1977), Scand. J. Clin. Lab. Invest. $37,563-566$

3. Morin, L. G. (1977), Clin. Chem. 23, 646-652

4. Thomson, W. H. S. (1969), Clin. Chim. Actta 23, 105-120
Stadt Nürnberg - Klinikum Dr. G. Weidemann Institut für Klinische Chemie Postfach 8500 Nürnberg 15 


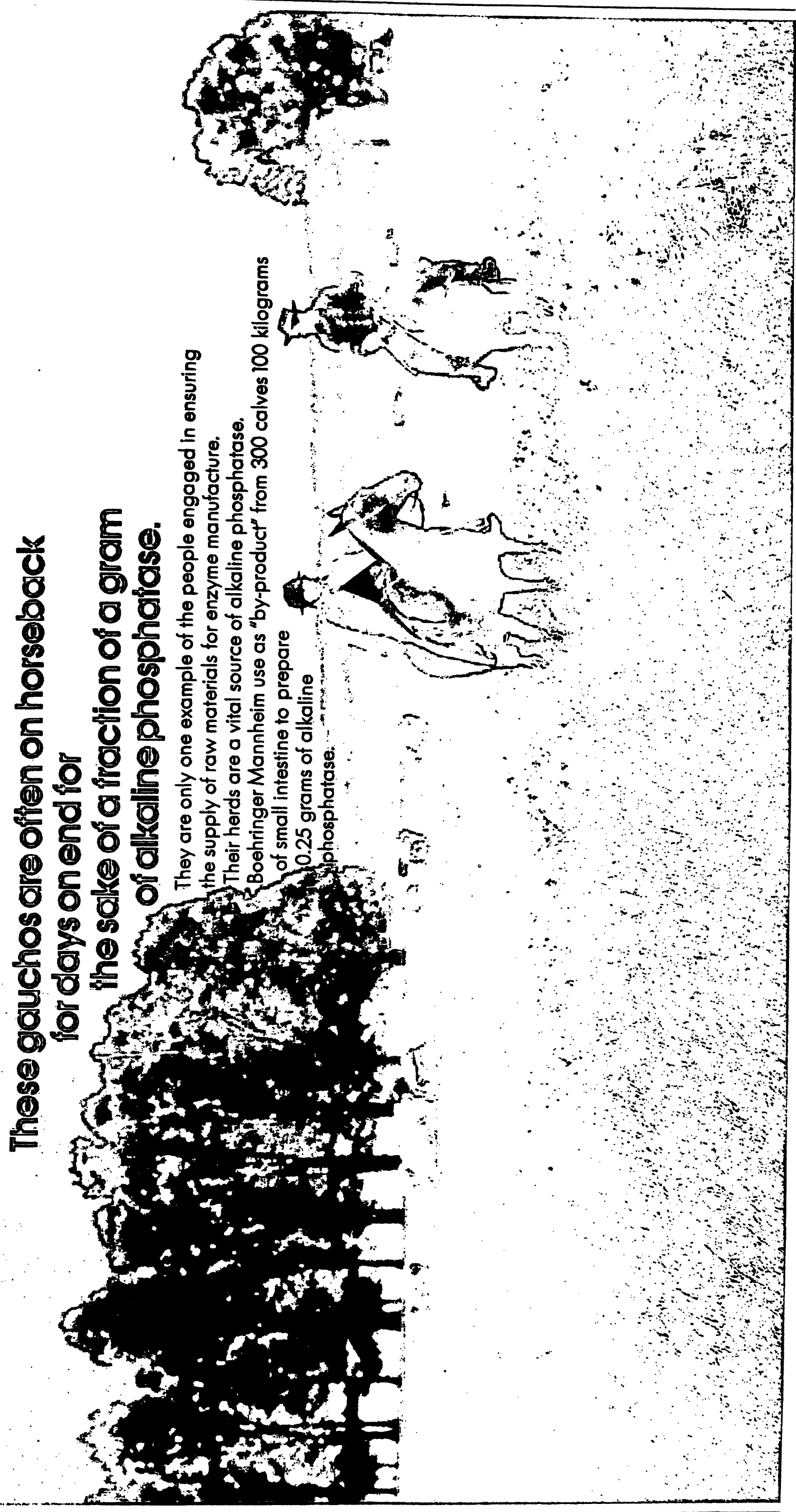


Eberhard Hofmann

\section{Funktionelle Bio-} chemie des Menschen

Band 1 und Band 2

1979. 642 S. mit 109 Abb. $11 \times 18$ (Reihe Wissenschaft). Gbd. 38.- DM

Das Buch faßt das derzeitige Wissen um die vielfältigen biochemischen Vorgänge im menschlichen Organismus zusammen. Es stellt die Biochemie unter funktionellen Gesichtspunkten dar und verknüpft dabei den klassischen Stoff der Physiologischen Chemie wie Blut, Körperflüssigkeiten. Hormone. Ernährung u. a. mit den modernsten Erkenntnissen der Molekularbiologie zu einem interdisziplinären Werk.

Ausführliche Informationen erhalten Sie in Ihrer Buchhandlung oder Schreiben Sie an den Verlag (Postfach 5829. 6200 Wiesbaden 1).

Friedr. Vieweg \& Sohn Verlagsgesellschaft mbH Braunschweig/Wiesbaden

\section{Vitamin $\mathrm{B}_{\mathbf{1 2}}$}

Proceedings of the Third European Symposium on Vitamin $B_{12}$

Editors

B. Zagalak, W. Friedrich

$1979.17 \mathrm{~cm} \times 24 \mathrm{~cm}$.

Approx. 1100 pages. Hardcover.

Approx. DM 190,-; \$112.00

ISBN 3110076683

\section{Biochemistry of Wounded Plant Tissues}

Edited by Dr. Günter Kahl, Professor for Botany, Department of Biology, Johann Wolfgang Goethe University of Frankfurt am Main. $1978.17 \mathrm{~cm} \times 24 \mathrm{~cm} .684$ pages. Hardcover DM 180.00 ISBN $311006801 \mathrm{X}$

This volume comprises original studies and survey articles by competent scientists from world-famous laboratories. It offers an overview of the present state of knowledge as regards the biochemistry and molecular biology of wound processes and woundhealing mechanisms as based on model systems from the plant kingdom. Extensive bibliographies of most recent literature are appended to each article, making the volume a rich source of information. 